Abstract

\title{
Algebraic Solutions in Scalar Field Cosmology: Reconstruction of the Dark-Energy Equation of State and the Inflationary Potential ${ }^{+}$
}

\author{
Andronikos Paliathanasis ${ }^{1,2}$ \\ 1 Instituto de Ciencias Fisicas y Matematicas, Universidad Austral de Chile, Valdivia 5090000, Chile; \\ anpaliat@phys.uoa.gr \\ 2 Instituto of Systems Science, Durban University of Technology, PO Box 1334, Durban 4000, South Africa \\ + Presented at Symmetry 2017-The First International Conference on Symmetry, Barcelona, Spain, 16-18 \\ October 2017.
}

Published: 4 January 2018

An algebraic solution for arbitrary potential is presented in the context of scalar field cosmological models. That result is used to generate new solutions of the scalar field equations in homogeneous and isotropic universes. A series of generalizations of the Chaplygin gas and bulk viscous cosmological solutions for inflationary universes are found. Finally, we show how the Hubble slow-roll parameters can be calculated using the solution algorithm and we compare these inflationary solutions with the observational data provided by the Planck 2015 collaboration to constraint and rule out some of these models.

(C) 2018 by the author. Licensee MDPI, Basel, Switzerland. This article is an open access article distributed under the terms and conditions of the Creative Commons Attribution (CC BY) license (http://creativecommons.org/licenses/by/4.0/). 